\title{
Response surface optimization for hot air-frying technique and its effects on the quality of sweet potato snack
}

\begin{abstract}
Response surface methodology was employed to obtain the optimum process conditions for frying sweet potato (Ipomoea batatas) via hot-air frying technique. Sweet potato samples were fried at different frying temperature $\left(140-160^{\circ} \mathrm{C}\right)$ and time $(4-12 \mathrm{~min})$. These variables were used as independent variables whose effects on fat and moisture contents; hardness and color were evaluated. The experimental results were fitted with a second-order polynomial equation by a multiple regression analysis. From the response surface methodology analysis, the recommended frying condition from the study was found to be $150^{\circ} \mathrm{C}$ for $12 \mathrm{~min}$. Optimized sweet potato samples were compared with deep fat-fried samples; where the former had very low fat content i.e. decreased by $90.1 \%$, lighter color and harder texture. Panellist selected the optimized air-fried samples for several sensory attributes which relates to quality. Frying sweet potatoes via hot air-frying technique can be considered as a healthier alternative to prepare fried food for consumption.
\end{abstract}

